Mots. Les langages du politique

\title{
Fin de parti(e). La dénomination du PS en questions
}

End of (the) party. The name of the PS in questions

Fin del partido. El nombre del PS en cuestión

Éric Treille

\section{(2) OpenEdition}

\section{Journals}

Édition électronique

URL : https://journals.openedition.org/mots/25225

DOI : $10.4000 /$ mots.25225

ISSN : 1960-6001

\section{Éditeur}

ENS Éditions

\section{Édition imprimée}

Date de publication : 11 juillet 2019

Pagination : 73-90

ISBN : 979-10-362-0170-7

ISSN : 0243-6450

\section{Référence électronique}

Éric Treille, «Fin de parti(e). La dénomination du PS en questions », Mots. Les langages du politique [En ligne], 120 | 2019, mis en ligne le 01 janvier 2022, consulté le 23 avril 2022. URL : http:// journals.openedition.org/mots/25225; DOI : https://doi.org/10.4000/mots.25225 


\section{Éric Treille}

\section{Fin de parti(e). \\ La dénomination du PS en questions}

C'est une des conséquences inattendues de l'élection présidentielle de 2017 : l'onomastique partisane française en est sortie profondément transformée (Offerlé, 2018 [1987], p. 116-122 ; Bacot, 2018a). De la création d’En Marche!, devenu La République En Marche !, et de La France insoumise, issue du Parti de gauche, à l'abandon du nom Front national pour celui de Rassemblement national ${ }^{1}$, quasiment aucune formation politique n'est sortie indemne de ce scrutin hautement «disruptif» (Perrineau éd., 2017).

Comme ses concurrents, le Parti socialiste n'a pas échappé à ces batailles de dénomination. Benoît Hamon a quitté la «vieille maison » pour fonder le Mouvement du 1er juillet ou M1717, une formulation lexico-syntaxique associée à une date, ensuite rebaptisé Génération.s. Le groupe parlementaire du PS à l'Assemblée nationale a porté pendant un peu plus d'un an le nom Nouvelle gauche en remplacement de l'appellation Socialiste, écologiste et républicain, avant de reprendre la dénomination historique de Socialistes et apparentés. Plus important encore, le nom du Parti socialiste s'est invité dans les débats du congrès d'Aubervilliers des 7 et 8 avril 2018, Stéphane Le Foll, candidat à la tête du parti, ayant proposé de troquer la dénomination PS contre celle de Les Socialistes, une modification morphologique mimétique à celle déjà engagée parl'Union pour un mouvement populaire, devenue Les Républicains en 2015.

Ces interrogations sur le nom du PS ne sont cependant pas nouvelles : elles apparaissent au contraire comme un quasi-invariant pour un parti sommé de transformer son identité politique après chaque défaite électorale. En 1993, Michel Rocard avait inauguré les états généraux de la gauche en posant directement la question du changement de nom du PS et de l'abandon du terme

1. Séparée depuis la signature du programme commun en 1972, la famille radicale a décidé le 9 décembre 2017 de se réconcilier et d'adopter une nouvelle dénomination : le Mouvement radical, social et libéral. La fusion entre le Parti radical de gauche et le Parti radical dit «valoisien » a cependant été de courte durée, la grande majorité des cadres du PRG faisant le choix de retourner à leur formation d'origine en raison de dissensions sur la stratégie à adopter lors des élections européennes de 2019.

Arènes (Université de Rennes - EHESP - CNRS)

eric.treille@laposte.net 
socialiste, prolongeant ainsi le travail préalable déjà effectué pour distribuer de façon anticipée des «certificats de déqualification » et de «désappropriation du sigle socialiste » au profit de l'Alliance des Français pour le progrès pendant la campagne des législatives (Treille, 2001 ; Cépède, 1996 et 1994). En 2002, Arnaud Montebourg, Vincent Peillon et B. Hamon, les principaux opposants à François Hollande, s'étaient mis dans les pas des premiers fondateurs du PS en prenant pour nom de motion Nouveau Parti socialiste, réminiscence du nom du parti à l'issue du congrès d'Alfortville de mai 1969.

De 1993 à 2018, les batailles sur la dénomination du PS ont en revanche différé sur plusieurs points. Les états généraux de la gauche ont répondu à la défaite historique des élections législatives du 21 mars 1993. Marqué également par la chute du mur de Berlin et la transformation transitoire du Parti communiste italien en «cosa» (la «chose») puis finalement en Parti démocrate de la gauche (Dormagen, 1995; Lazar, 1992), le PS avait mis en débat le terme même de socialiste dans un processus d'introspection interne. En 2018, les différents candidats à la tête du PS ont fait campagne dans un parti fragilisé par le résultat historiquement faible de son candidat à l'élection présidentielle $-6,35 \%$ - et l'absence de leadership interne ${ }^{2}$. En soumettant à la question la référence à la notion de parti, ils ont non seulement prolongé les choix déjà adoptés par Emmanuel Macron et Jean-Luc Mélenchon, mais ils ont également interrogé le sens à donner à leur organisation et aux pratiques militantes en son sein. De même, ils se sont inscrits dans un processus plus large de délégitimation de l'appellation partisane engagé par la mise en œuvre de primaires - citoyennes en 2011 et de la Belle Alliance populaire en 2017 (Lefebvre, Treille éd., 2019 et 2016) -, nouvelles procédures de désignation qui ont imposé à chacun des candidats de dépasser la forme partisane socialiste.

Alors que les débats sur le nom du PS ont longtemps fait partie des modes privilégiés par cette formation politique pour gérer ses crises identitaires postdéfaites électorales, la réactivation répétée de cette "grammaire de l'institution partisane » souffre aujourd'hui « d'usure » (Lefebvre, 2018). Le parti d’Épinay a conservé son nom et, dans le même temps, a vendu son siège historique du 10 rue de Solférino - et par la même occasion abandonné un emblématique substitut nominatif -, a décidé de ne pas conduire de liste sous ses propres couleurs lors du scrutin européen de 2019 - et ce pour la première fois depuis 1979 - ou encore a éteint un à un ses rituels, de la tenue de son université d'été

2. À la différence du Rassemblement bleu Marine et plus encore d’En Marche !, dont l'acronyme a obéi à une logique de siglaison anthroponyme reprenant l'ordre lettrique des prénom et nom de son fondateur, l'absence de leaders en capacité de nommer un courant ne permet pas aux militants socialistes, ni aux journalistes, d'utiliser de substituts au sigle PS. Ainsi, il n'existe pas de fauristes, de le folliens - ou de maureliens avant qu'Emmanuel Maurel ne quitte le PS - pour remplacer les termes de hollandais, de vallsistes et d'hamonistes, ou encore de fabiusiens et de jospinistes ou, plus loin dans le passé, de mitterrandistes et de rocardiens. 
de La Rochelle après vingt-trois ans d'existence3 à l'ascension de la colline de Solutré, un lieu majeur de la mémoire mitterrandienne.

Dans ce cadre, nous souhaitons analyser la façon dont ces souhaits non aboutis de rebaptiser le PS ont agi à la fois comme des symptômes et comme des remèdes rhétoriques à la défaite de 2017. Nous proposons de revenir sur ce que ces luttes internes, dans leurs continuités comme dans leurs ruptures, nous disent d'un parti « rétréci » (Lefebvre, Sawicki, 2006) pour lequel l'attachement à son sigle - et au capital symbolique qui lui est lié - est encore une manière d'authentifier, à l'image du Parti radical ou encore du Parti communiste français (Pudal, 2009), son ancienne domination sur la gauche et plus largement sur le jeu politique de la Ve République.

On s'efforcera alors de mettre en valeur les confrontations entre les tenants du changement de nom et les gardiens de la «vieille maison » lors du congrès d'Aubervilliers, puis le déplacement des propositions visant l'abandon de la référence au socialisme à celles ciblant la démonétisation du terme de parti, les invitations au «dépassement du PS» dans la primaire de la Belle Alliance populaire et enfin la «bataille de Solférino » ou la décision du PS de quitter son siège parisien pour rejoindre la ville communiste d'Ivry-sur-Seine.

\section{Les Socialistes versus Les Républicains}

Après le temps des épreuves et de l'échec politique, le $78^{\mathrm{e}}$ congrès du Parti socialiste, organisé à Aubervilliers en avril 2018, devait être pour le PS le symbole de son renouveau. Ce premier rendez-vous militant depuis l'élection d'E. Macron à la présidence de la République a surtout été celui de la fidélité au passé, au risque de la nostalgie. En brandissant l'ensemble de ses cartes d'adhérents depuis le congrès fondateur d’Épinay de 1971 comme autant de «trophées » de vie militante (Cépède, Almeida, 2007, p. 97), Louis Mexandeau, 86 ans, en a été le héros, éclipsant même le nouveau premier secrétaire du PS, Olivier Faure. Le plus écouté et le plus applaudi des orateurs invités à s'exprimer, l'ancien ministre de l'Industrie et de la Recherche chargé des PTT sous François Mitterrand, a scellé en convoquant ces marqueurs identitaires le sort d'un des enjeux du congrès : le PS devait-il, à l'image de ses concurrents, changer de nom? En revendiquant ainsi la fierté d'être socialiste et d'appartenir au PS depuis sa refondation, L. Mexandeau avait posé le décor de son parti : celui de la stabilité lexicale. Le PS allait conserver son label d’organisation.

3. Le PS n'organise plus d'université d'été pour ses militants depuis 2016, mais un séminaire de formation tenu à huis clos pour ses seuls élus. Son premier secrétaire, Olivier Faure, a cependant promis d'ouvrir à nouveau le rendez-vous de rentrée de La Rochelle à l'ensemble des adhérents. 
L'abandon de la siglaison PS comme procédé morphologique a pourtant été inscrit à l'agenda du congrès d'Aubervilliers par l'un des principaux candidats au poste de premier secrétaire. Le 25 août 2018, S. Le Foll, ancien ministre de l'Agriculture et porte-parole du gouvernement de Manuel Valls, a ainsi proposé sur l'antenne de RTL de «changer le nom du Parti socialiste. Ma proposition : Les Socialistes». Interrogé par le journal Le Monde, ce très proche de F. Hollande a précisé sa pensée : «il y a Les Républicains, Les Verts... Il y a même Les Constructifs! Je pense que s’appeler Les Socialistes, ça sera aussi une manière d'être présents, d'exister parce que ce nom est important et d'avoir un changement qui permet d'ouvrir le parti » (Le Monde, 25 août 2018).

En appelant à une formulation "plus simple, plus claire», S. Le Foll rompait ainsi avec la solution d'abrègement typographique choisie lors du congrès d’Épinay, mais également avec l'histoire plus longue des dénominations des partis représentant la France au sein de l'Internationale socialiste depuis 1905 (Cépède, 2017). En passant du singulier (le Parti socialiste) au pluriel (Les Socialistes), ce choix prenait également acte des clivages qui avaient surgi pendant la campagne présidentielle de 2017 et proposait en réponse une démarche synthétique effaçant les départs successifs des deux finalistes de la primaire, $M$. Valls et B. Hamon, comme les nombreuses indisciplines qui avaient émaillé le quinquennat de F. Hollande (Sawicki, 2017). En cela, ce débat sur le nom du PS a souligné le rôle clé des scissions et des exclusions4 dans les stratégies dénominatives du parti. Léon Blum avait ainsi maintenu le nom de la Section française de l'Internationale ouvrière après les départs des néo-socialistes en 1933 et des pivertistes en 1938 (Cépède, 2017).

En exhumant du passé récent du PS un schème de dénomination déjà brièvement utilisé en 2006 au moment où le PS avait ouvert son mode de recrutement militant en lançant des adhésions à 20 euros (Barboni, Treille, 2010), S. Le Foll engageait également son parti dans la voie du mimétisme dénominatif, le PS étant invité à adopter le même procédé que l'UMP, devenue LR. Parti défait, le PS n'est plus depuis l'élection présidentielle de 2017 une formation politique dominante. Dépassé par J.-L. Mélenchon et LFI, il n'est même plus le premier parti de la gauche. Dans ce cadre, l'abandon du sigle PS pour la dénomination Les Socialistes devait permettre à la fois de désigner plus fortement encore l'orientation politique d'un parti marqué par les évolutions doctrinales de la présidence de F. Hollande (Rioufreyt, 2016) et de s’imposer comme le pendant à gauche, par construction symétrique, des Républicains, malgré le déséquilibre des résultats électoraux présidentiels, puis législatifs. En se posant ainsi comme l'alter ego langagier du principal parti de la droite - non sans avoir dénoncé à l'époque le choix de cette captation du champ lexi-

4. Voir sur ce sujet l'entretien accordé par Emmanuel Maurel au journal Le Monde intitulé «Ce n'est pas un départ, c'est une scission » pour expliquer les raisons de sa rupture avec son parti (Le Monde, 13 octobre 2018). À ce propos, voir aussi Bacot, 2018b. 
cal de la République -, le PS souhaitait s'auto-accorder un brevet d'authenticité politique dans un jeu hautement concurrentiel et ranimer verbalement un bipartisme qui a disparu depuis l'émergence du FN (Haegel, 2002), puis de LFI et d'EM (Dolez, Fretel, Lefebvre éd., 2019).

Contre toute attente, cette proposition reçut l'assentiment du premier secrétaire du PS. Après avoir toujours refusé de débattre de ce sujet, JeanChristophe Cambadélis a affirmé que le nom « [serait] sur la table» (Le Figaro, 22 mai 2017). À l'AFP, le premier secrétaire démissionnaire précise :

Le PS ne peut vivre comme la SFIO avec une verticalité, ça, nous le laissons à JeanLuc Mélenchon, à Emmanuel Macron, à Marine Le Pen. Aujourd'hui, il faut faire vivre plus fortement les mouvements associatifs, être en interaction avec la société civile, les mouvements citoyens. Il faut trouver une nouvelle formule d'agrégat politique. (AFP, 22 mai 2017)

Sur son compte Twitter, il ajoute : «il y a une formulation du progressisme à inventer». Puis, toujours le même jour : «il faudra marquer une nouvelle époque qui peut passer par un changement de nom, un changement de lieu ».

L'inscription tardive du congrès d'Aubervilliers dans le calendrier politique des adhérents socialistes a cependant empêché la tenue d'un véritable débat sur le nom du PS. Délibérément repoussé au mois d'avril 2018, soit près d'un an après la défaite de $B$. Hamon à l'élection présidentielle, ce rendez-vous militant n'a pas eu pour objet de faire l'inventaire du quinquennat ou encore de remettre en question la doctrine du parti. Le seul véritable enjeu de ce congrès d'apaisement a été la désignation d'un nouveau premier secrétaire et de la direction du parti. Dans ce cadre, la question de la labellisation organisationnelle ne s'est pas posée. Les candidats comme les militants ont préféré jouer la stabilité nominative, considérant qu'un changement trop brusque pouvait affecter une marque politique encore fragilisée par le résultat de son représentant à l'élection présidentielle et la forte diminution de son groupe parlementaire à l'Assemblée nationale.

Une autre raison plus circonstanciée a pu également expliquer l'insuccès de la proposition d'abandon du nom PS de S. Le Foll : Marie-Noëlle Lienemann, sénatrice de Paris et alors coresponsable du courant «L'Union et l'espoir » avec Emmanuel Maurel, est détentrice de la marque Les Socialistes auprès de l'Institut national de la propriété industrielle depuis janvier 2016. Comme l'a souligné cette ancienne frondeuses, "ça tombe mal pour eux». "Tant que le mot socialiste reste dans le nom du parti, ça me convient. Mais le changement de nom en "Les Socialistes", ça ne doit pas seulement être une initiative de S. Le Foll. Il faut que l'idée soit partagée et acceptée par les membres du bureau national du PS» (Le Parisien, 25 août 2017). Elle a également ajouté qu'elle avait déposé

5. M.-N. Lienemann et E. Maurel ont depuis créé leur propre parti : la Gauche républicaine et socialiste (GRS). 
le nom à l'INPI « lorsque Valls et compagnie pensaient qu'il ne fallait plus être socialiste » (Le Point, 25 août 2017), « ne voulant pas que son parti abandonne le terme de socialiste» (Le Parisien, 25 août 2017).

\section{Manuel Valls et La Gauche}

En affirmant que le nom du PS « [serait] sur la table», J.-C. Cambadélis a opéré un véritable tournant. Quelques mois auparavant, le premier secrétaire se refusait pourtant à tout changement d'appellation. Fortement invité à réinventer la dénomination de son parti par le Premier ministre, Manuel Valls, avant l'engagement dans la bataille présidentielle, il avait déclaré qu’il était «favorable à changer la carte d'identité du Parti socialiste [...] mais pas au changement de nationalité » de son parti (Le Huffington Post, 22 mai 2017).

Il est vrai que l'exercice onomastique proposé par M. Valls n'était pas de même nature que celui suggéré par S. Le Foll. Contrairement au projet de l'ancien ministre de l'Agriculture d'engager son parti dans une stratégie mimétique avec LR, le Premier ministre n'a pas souhaité conserver le mot socialiste, même sous le titre générique Les Socialistes. Alors que J.-C. Cambadélis avait déclaré «j'ai tendance à penser : le problème est moins socialiste que parti», M. Valls avait choisi de cibler avant tout ce marqueur identitaire.

Cette proposition de «disqualification» du socialisme n'est cependant pas neuve (Pudal, 2002, p. 547). Depuis 2007, M. Valls a tenté d'imposer ce débat au sein de son parti, rappelant que la «permanence des sigles cache des phénomènes de discontinuités» (Ethuin, Lefebvre, 2015, p.10). Pour le maire d'Évry, l'enjeu était moins d'engager sa formation dans une nouvelle stratégie dénominative que de montrer les limites, selon la terminologie de Saul Kripke, d'un «désignateur rigide» (Offerlé, 2018 [1987], p.117) pour traduire les différents changements idéologiques opérés par le PS depuis 1981 dans son appellation. Après la défaite de Ségolène Royal en 2007, il a ainsi proposé d'" entrer dans un processus de refondation qui peut même mener à un changement de nom du PS. Le mot de gauche est le plus beau qu'on puisse aujourd'hui porter. C'est celui qui représente de nouveau une espérance». Puis, il a affirmé vouloir «tout remettre en cause » jusqu'au nom de son parti : «Parti socialiste, c’est daté. ça ne signifie plus rien. Le socialisme, ça a été une merveilleuse idée, une splendide utopie. Mais c'était une utopie inventée contre le capitalisme au XIx siècle ${ }^{6}$. En 2009 , invité dans le Journal de 20 heures de France 2, il a également déclaré : "si nous voulons demain rassembler des énergies, des intellectuels, des chercheurs, des ouvriers qui ne votent plus pour nous, il faut créer une grande organisation politique autour

6. M. Valls, Pour en finir avec le vieux socialisme... et être enfin de gauche! Entretiens avec Claude Askolovitch, Paris, Robert Laffont, 2008. 
de ce mot merveilleux qui est celui de la gauche car je le crois plus moderne» (France 2, juin 2009). M. Valls a réaffirmé que « le socialisme, c'est une idée du XIXe siècle». En 2012, à nouveau sollicité par France 2, il a répondu à l'interrogation de David Pujadas : « socialiste? - Peu importe les mots, ma famille, c'est la gauche. Ma famille, c'est une gauche efficace qui gouverne aujourd'hui » (France 2, décembre 2012). En 2014, dans un long entretien accordé à L'Obs, il a proposé la dénomination «maison commune de toutes les forces progressistes », jugeant le mot socialiste « dépassé » (L’Obs, le 23 décembre 2014). Le premier secrétaire, J.-C. Cambadélis, lui a alors répondu : «je reste sur la position de F. Mitterrand. Socialiste est un beau nom qui a fait ses preuves, autant le garder " (L'Obs, le 23 décembre 2014). Le président de l'Assemblée nationale, Claude Bartolone, est allé plus loin encore en qualifiant la proposition de M. Valls de "faute» (RTL, le 23 décembre 2014). En 2017, le député d’Évry relance cependant le débat en demandant que son parti entre «dans un processus de refondation qui peut même amener à un changement de nom » et suggère à nouveau le nom de «gauche» (L'Express, le 25 août 2017).

La proposition de S. Le Foll ne s'est pas inscrite dans le même cadre de transformation. Contrairement à M. Valls ou, avant lui, à Laurent Fabius, qui avait émis l'idée de transformer le nom de Parti socialiste en Parti social-démocrate, ou, encore plus loin dans le temps, à Gaston Defferre, qui avait lancé dans les années 1960 un débat sur la transformation du nom de sa formation en Parti socialiste démocratique ou Parti démocratique socialiste, le terme socialiste est ici clairement assumé. À l'image des autres formations politiques françaises, le travail d'onomastique proposé par l'ancien ministre de l'Agriculture visait avant tout le parti comme « forme organisationnelle et non comme expression d'une doctrine» (Sawicki, 1996).

En cela, S. Le Foll n'innove pas. L'appellation Les Socialistes condense en fait deux évolutions déjà anciennes. La première est relative à la disparition du label partisan. À la suite des partis de droite, qui ont historiquement eu des réticences à user du mot parti comme dénomination organisationnelle, préférant des termes plus neutres comme mouvement, union ou encore rassemblement, des partis de gauche comme LFI ont également entrepris de gommer le lexique partisan de leur sigle ou de leur dénomination. La seconde tient compte des rapports de concurrence existant entre les différentes formations politiques. S. Le Foll ne propose pas seulement la disparition du mot parti, il invite également les membres de son mouvement à épouser la «bonne» vision des partis politiques (Leca, Offerlé, 1988, p. 54) mise en œuvre par l'UMP lorsqu'elle a changé de nom et de statut pour se transformer en Les Républicains le 28 mai 2015.

Dans le contexte mouvant de l'après-élection présidentielle dominé par l'irruption de LFI et d'EM, la demande de rattachement du PS à une « famille politique » identifiable, au-delà de sa seule forme sténographique, n'est cependant pas une opération de réétiquetage isolée. Lors de son séminaire de rentrée 
du 24 juin 2017 à la Maison de la chimie, un nouveau visuel Socialistes! avait déjà été utilisé7. Depuis 2015, à la siglaison PS se trouve également accolé le terme social-écologie. Surtout, le groupe socialiste de l'Assemblée nationale a abandonné le nom de Socialistes, écologistes et républicains pour la nouvelle dénomination de Nouvelle gauche ${ }^{8}$. Pour la première fois depuis 1958, le groupe socialiste ne faisait pas référence au socialisme. Pour justifier ce changement de dénomination, son président 0 . Faure avait alors déclaré : « le qualificatif "socialiste" ne sera désormais présent que dans la déclaration politique du groupe " 9 . Ce changement fut cependant de courte durée. Lors des journées parlementaires des 10 et 11 septembre 2018, les trente élus socialistes de l'Assemblée nationale décident de rebaptiser leur groupe Socialistes et apparentés, renouant ainsi avec une dénomination historique. Selon O. Faure, «Nouvelle gauche avait l'immense inconvénient de ne pas permettre l'identification entre le Parti socialiste et son propre groupe parlementaire alors qu'il en est le relais naturel » (Le Monde, le 10 septembre 2018).

Les « ruptures politiques» ne génèrent donc pas nécessairement des « redénominations en masse» (Guillorel, 2012). Même si son groupe à l'Assemblée nationale n'a pas porté pendant un peu plus d'un an ce qualificatif politique, le PS a échappé au changement de son nom et à l'impératif de «faire parti autrement» (Ethuin, Lefebvre, 2015, p.10), malgré la demande d'un candidat au premier secrétariat de son parti et du soutien de J.-C. Cambadélis. Le format adopté par le congrès d'Aubervilliers y a été pour beaucoup. Programmé tardivement et à des fins essentiellement thérapeutiques, ce rendez-vous interne n'a pas inscrit parmi ses chantiers prioritaires la définition d'une nouvelle stratégie dénominative. En revanche, la forme organisationnelle du parti a suscité de nombreuses interrogations, au-delà de la siglaison même du nom du PS. Comme S. Le Foll, O. Faure a émis des propositions dans son texte de motion visant à mettre en place un « parti plate-forme ». Il a ainsi, sitôt élu par les adhérents, proposé d'organiser des moments militants ouverts aux sympathisants qui auraient alors la possibilité de débattre et de voter contre un euro symbolique. Ce nouveau droit leur permettrait ensuite d'être intégrés dans le corps électoral d'une primaire que pourrait à nouveau organiser le PS, un dispositif de désignation du candidat socialiste à l'élection présidentielle désormais

7. Cet usage de la ponctuation n'est pas isolé. Valérie Pécresse, E. Macron, S. Le Foll et François de Rugy ont également mis à contribution le point d'exclamation pour signer leurs mouvements respectifs : Libres!, En Marche! La République En Marche!, Hé oh la gauche! et Écologistes!.

8. Le nom de Nouvelle gauche n'est pas une appellation inédite. Il a déjà été utilisé par B. Hamon lorsqu'en 1993 il créa avec ses proches, dont O. Faure, son propre courant - néo-rocardien - au sein du MJS avant de fondre son organisation au sein du NPS.

9. «Nous sommes socialistes, nous sommes écologistes, nous sommes démocrates, nous sommes républicains, nous sommes progressistes et nous pouvions avoir cette addition dans le nom. Nous avons cherché à vous simplifier la tâche, pour la presse écrite et aussi pour la presse audiovisuelle. Nous avons choisi de nous appeler plus simplement groupe de la Nouvelle gauche avec l'idée de rappeler ce que nous sommes» (Le Parisien, le 27 juin 2017). 
logé au cœur même de la nomenclature des marques politiques de la gauche française (Lefebvre, Treille éd., 2019).

\section{Le dépassement du PS dans la primaire de la Belle Alliance populaire}

S. Le Foll et M. Valls n'ont pas été les seuls responsables socialistes à porter l'idée d'un changement de nom du PS : Julien Dray, membre du bureau national, a également réclamé une transformation onomastique de son parti pour répondre au sévère échec électoral de la gauche aux élections régionales de 2015 et à la perte emblématique des régions île-de-France, Rhône-Alpes, NordPas-Calais et PACA.

On va essayer de faire que la gauche soit au second tour de l'élection présidentielle donc qu'elle se regroupe, qu'elle se rassemble d'une manière ou d'une autre pas seulement par souci défensif, elle doit le faire en travaillant, en étant adossée à un projet. On va changer beaucoup de choses dans le fonctionnement de notre parti. On a dit qu'on allait même changer de nom vraisemblablement. (Le Lab politique, lelab.europe1.fr, 13 décembre 2015)

En réponse, J.-C. Cambadélis réactive la proposition déjà formulée en 2014 par M. Valls de fondation d'une «maison commune de toutes les forces progressistes» en prônant la création d'un nouveau " grand mouvement progressiste » construit autour de la formation socialiste : «le changement de nom du PS n'est pas à l'ordre du jour. Le dépassement du PS l'est! (message sur Twitter, le 13 décembre 2015).

Cette stratégie visant à la mise en place d'une «nouvelle alliance» en vue de l'élection présidentielle de 2017 n'est pas inédite (Lefebvre, 2017). Après avoir été déjà utilisée une première fois par S. Royal en $2009^{10}$, elle avait été évoquée par J.-C. Cambadélis le jour de son élection à la tête du PS en mai 2015, puis exprimée, quasi mot pour mot, par le Premier ministre lors de l'université d'été de La Rochelle en août 2015: «organisons ce dépassement de ce qui nous différencie pour nous rassembler ». La fragmentation du jeu politique à gauche lors des élections régionales et le risque de ne pas pouvoir figurer au second tour de l'élection présidentielle ont cependant donné un sens nouveau à ces appels à l'unité de la gauche et au souhait du premier secrétaire de réaliser un «nouveau congrès d'Épinay », c'est-à-dire « la substitution au PS d'une formation politique rassemblant tous ceux qui auront participé».

Les ambitions affichées en 2016 sont cependant beaucoup plus mesurées. Les projets d'une «grande convention de l'alliance populaire» et d'adoption

10. S. Royal a plaidé pour le «dépassement du PS» lors de la fête de la Fraternité de son association Désirs d'avenir à Montpellier le 19 septembre 2009. 
d'un «schéma directeur pour la prochaine présidentielle » sont remisés et remplacés par la proposition d'une alliance politique moins contraignante et à visée purement électorale : il s'agit de trouver un moyen de surmonter la division de la gauche dans le tripartisme et d'éviter un nouveau 21 avril ; un moyen également de permettre à un F. Hollande, fortement contesté par son propre camp, de briguer un second mandat (Sawicki, 2017).

Contrairement à la droite qui s'était engagée, en novembre 2002, dans la création de l'UMP, fusion de Démocratie libérale et du RPR (Haegel, 2002), le PS n'a donc pas fait le choix de la refondation partisane interne ou de redénomination. J.-C. Cambadélis a préféré plaider pour la mise en place d'une « alliance populaire » en vue de l'élection présidentielle de 2017, une simple « fédération de partis» et non un "grand mouvement progressiste» comme envisagé en 2015. Malgré ce format peu ambitieux, cette nouvelle offre partisane est cependant boudée, aussi bien par le PCF, le PG que par Europe-Écologie-les-Verts, Cécile Duflot exprimant sa préférence pour une «coalition de transformation» en lieu et place de ce qu'elle a appelé une «coalition du prétendu "cercle de la raison" " (Le Monde, le 15 décembre 2015).

Le projet de primaire de coalition porté par le journal Libération en janvier 2016 a permis au PS de relancer involontairement son projet de dépassement sous le nouveau nom de Belle Alliance populaire (Lefebvre, Treille éd., 2016). Pour contrer cette initiative, le premier secrétaire du PS a d'abord exigé une primaire large de «Macron à Mélenchon ». Puis, à la suite des acceptations de participation conditionnelle du PCF comme d'EELV - F. Hollande doit être exclu du scrutin - et de la candidature de J.-L. Mélenchon à l'élection présidentielle, il a concédé le déclenchement de son «innovation démocratique » (Lefebvre, Treille, 2018) en définissant un mode de présélection volontairement a minima, ajusté prioritairement aux intérêts du président en exercice, dans son calendrier comme dans son format.

Faute de partenaires - le PCF soutient la candidature de J.-L. Mélenchon à l'élection présidentielle; EELV organise sa propre procédure de sélection-, la primaire de la Belle Alliance populaire est alors réduite au PS et à ses alliés les plus proches : le PRG, l'Union des démocrates et des écologistes, le parti écologistes! et le Front démocrate, écologiste et socia ${ }^{11}$. Ambitieusement présentée comme une "fédération de la gauche de transformation », cette alliance électorale a surtout eu pour objectif de desserrer l'entre-soi socialiste et de conjurer la multiplication des candidatures socialistes en dehors du parti, et moins de se transformer en parti en tant que tel - la BAP - ou de développer de nouveaux réseaux militants, la primaire étant ouverte à tous les sympathisants.

11. Jean-Luc Bennahmias a nommé cette alliance "maison commune des progressistes» en avril 2015, reprenant la dénomination déjà proposée par M. Valls en 2014 : «bâtir une maison commune de toutes les forces progressistes». 
L'extrême division cellulaire de la gauche a paradoxalement constitué un atout pour rompre l'isolement politique du PS. Au lieu d'inventer un nouveau label partisan, le PS a préféré multiplier le nombre de formations politiques satellites autour de lui (Aldrin, 2007), occupant l'espace politico-topographique écologiste et centriste. En créant un cartel électoral temporaire au-delà de son seul cercle ${ }^{12}$, il a dilué le temps de la campagne son nom dans un système de coopération interpartisan plus large, même si le prix de cette stratégie fut de mettre en équivalence, voire de faire exister artificiellement des formations politiques aux histoires, au nombre d'adhérents ou encore aux modes d'organisation très différents les uns des autres ${ }^{13}$.

La mise en œuvre de la primaire de la Belle Alliance populaire a également eu pour conséquence d'enrichir par le bas l'onomastique des partis de gauche de micro-partis créés spécifiquement pour ce scrutin afin d'encadrer les mobilisations des différents candidats socialistes et qui, contrairement à Sylvie Pinel, Jean-Luc Bennahmias et François de Rugy, ne pouvaient s'adosser à des structures partisanes qui leur soient propres (Phélippeau, 2018; Treille, 2018). De fait, depuis 2006 et surtout 2011, les primaires ont transformé le cursus honorum (Offerlé, 2017) des prétendants au magistère suprême en abaissant fortement le coût d'entrée dans la compétition politique. Pour se mettre sur les rangs, il n'est plus besoin de fonder ou de prendre le contrôle d'un courant ou, plus exigeant encore, de prendre la tête de son parti : de simples parrainages - et, pour les candidats extérieurs au PS, une affiliation de son mouvement à la Belle Alliance populaire - suffisent, ce qui permet de s'affranchir de l'obligation de «s'écurialiser» (Cos, 2016).

L'inventivité partidaire a ainsi touché l'ensemble des candidats réels ou supposés de la primaire. E. Macron a annoncé le 6 avril 2016 la naissance d'EM, raison sociale de l'Association pour le renouvellement de la vie politique, encore présentée, à ce moment précis de la campagne, comme une «sorte de think tank ${ }^{14}$ et non comme un parti concurrent du PS (Treille, 2019). A. Montebourg a lancé son propre micro-parti baptisé Le Projet France dont le schème associe la dimension axiologique du programme du candidat. B. Hamon a déposé les statuts d'une formation nommée Elpis, nom qui, dans la mythologie grecque, désigne une jeune femme personnifiant l'espoir, une corne

12. En ouvrant la primaire de la Belle Alliance populaire à de nouveaux partenaires, le PS a cependant fragilisé le lien historique qui le liait au PRG. Après avoir participé aux premières réunions, le Parti radical s'est retiré de l'accord et a présenté sa présidente directement à l'élection présidentielle, puis dans un second temps, a finalement rejoint le cadre de la primaire.

13. Ce qui n'a cependant pas pu être le cas du Mouvement républicain et citoyen, de Nouvelle donne et du Mouvement des progressistes, ces partis s'étant inscrits trop tardivement à la procédure de désignation.

14. Le journal Le Monde a ainsi relaté l'annonce de la création d'En Marche ! : «C'est ce jour-là, entre deux portes, que le ministre de l’Économie confie au président, l'air de rien : "je fais un truc à Amiens le 6 avril (2016), je lance un mouvement de jeunesse, une sorte de think tank” » (Le Monde, $1^{\mathrm{er}}$ avril 2017). 
d'abondance entre les mains ${ }^{15}$. De son côté, F. Hollande n'a pas attendu l'ouverture officielle de la primaire pour se transformer d'aspirant putatif en prétendant subliminal. Pour cela, ses proches se sont activés : S. Le Foll a créé le mouvement Hé oh la gauche !, un site baptisé « Notre idée de la France » a été mis en ligne et l'association Démocratie 2012, utilisée lors de la précédente primaire, réactivée sans changer de nom.

En décidant de traduire en actes son invention de 2009, le PS n'a donc pas seulement respecté ses statuts, il a également organisé son désengagement dénominatif en créant la Belle Alliance populaire, un cartel électoral à usage unique destiné à élargir l'assise politique d'un président sortant en difficulté. Cette structure autonome du PS diffère en cela des labels de campagne, à l'image de l'Alliance des Français pour le progrès, label choisi en 1993 pour gommer le sigle $P S$ des affiches des candidats aux élections législatives, ou encore de la démarche de la gauche plurielle mise en œuvre par Lionel Jospin en 1995 (Fiala, Rennes, 2002). En choisissant ce mode de régulation du leadership partisan, le PS a aussi contribué à durcir juridiquement les associations de soutien aux candidats en lice qui, pour financer leur campagne (Treille, 2018), ont alors dû transformer le cadre souple des courants créés pour les seules compétitions intrapartisanes en micro-partis ouverts sur l'extérieur, même si leur organisation sommaire et épisodique les ont davantage apparentés à des «partis d'intérimaires » (Offerlé, 2018 [1987]) qu'aux formations dont ils étaient issus ou aux tendances dont ils étaient les émanations.

\section{La " bataille de Solférino » ou la vente d'un substitut discursif du PS}

La décision de conserver le sigle PS lors du congrès d'Aubervilliers comme l'échec de la Belle Alliance populaire n'ont cependant pas clos les débats internes sur la symbolisation du parti. En décidant de vendre son siège historique, sis 10 rue de Solférino dans le $7^{\mathrm{e}}$ arrondissement de Paris, pour rejoindre la commune d'Ivry-sur-Seine, ville dirigée par le PCF depuis 1925, la direction socialiste ne s'est pas séparée d'un toponyme comme un autre : elle a également abandonné le substitut discursif le plus médiatique de la dénomination PS.

En effet, en ne conservant que 29 sièges à l'Assemblée nationale, à l'issue du second tour des élections législatives du dimanche 18 juin 2017, en lieu et place de 295 élus en 2012, le PS n'a pas seulement connu son plus grave échec électoral depuis 1969, il a aussi selon les mots de son trésorier, Jean-François Debat, subi un «choc financier considérable» (Le Parisien, 17 juillet 2017), le montant des subventions publiques passant de 25 millions d'euros par an à

15. Au-delà des stricts commentaires politiques, le choix de cette dénomination fit également l'objet de nombreuses railleries sur les réseaux sociaux. 
7 millions. Pour amortir cette chute considérable des recettes, la direction du PS a alors engagé un important plan de redressement économique dont la décision la plus emblématique a été la vente de son siège ${ }^{16}$, un très bel hôtel particulier occupé depuis 1980 dont, par métonymie, le nom de la rue a rapidement désigné le PS, soit Solférino, ou encore, dans sa formulation raccourcie et plus militante, Solfé.

Ce glissement lexical n'est pas propre au PS. Avant leur fusion avec le PRG, les Radicaux dits «valoisiens» se dénommaient ainsi parce que le siège de ce parti était sis rue de Valois. De même, le PCF est communément appelé Place du Colonel-Fabien et le Rassemblement pour la République a longtemps porté comme substitut discursif le nom de la rue de Lille. Ce nom s'inscrit cependant dans l'histoire propre du parti d'Épinay. Longtemps domiciliée au 12 cité Malesherbes - aujourd'hui siège de la fondation Jean Jaurès -, l'adresse de l'ancienne SFIO déplaisait à F. Mitterrand. Le nouveau PS a alors déménagé place du Palais-Bourbon, puis en 1978 a occupé la rue de Solférino. En 1980, le 10 est devenu officiellement le siège du PS, qui l'a acheté en 1986. Depuis cette date, malgré des défaites - notamment celle de 1993 -, le PS n'a jamais quitté ce site, alors qu'à droite, l'Union des démocrates pour la République, devenue RPR puis UMP et enfin LR, n'a pas connu la même fixité immobilière, chaque nouveau nom appelant une nouvelle adresse parisienne. LREM a également déménagé trois fois en l'espace de deux ans, empêchant ainsi la création d'un lien toponymique fort avec le mouvement.

La double défaite électorale du PS en 2017 a scellé la fin de cette métonymie. J.-C. Cambadélis a été le premier à imputer l'échec politique du parti au nom du PS et à la localisation de son siège. Interrogé par Le Figaro sur les évolutions du PS après les élections présidentielles et législatives ("y compris le nom, le siège ? »), il répond : "à partir du moment où vous changez, on change tout. Il faudra marquer une nouvelle époque». Dans ce cadre, la motivation économique - apporter 55 millions d'euros à une formation politique en crise financière - est rapidement devenue seconde.

La vente du siège socialiste s'est alors transformée en enjeu caché du congrès d'Aubervilliers, le toponyme remplaçant la siglaison. La proposition d'un déménagement dans une ville communiste a d'abord été fortement dénoncée. Patrick Kanner, le président du groupe socialiste du Sénat, a ainsi déclaré : «je n'ai rien contre l'union de la gauche mais on aurait pu aller à Sarcelles, au moins nous aurions été chez nous ». Luc Carvounas, candidat au poste de premier secrétaire, a opté pour le même argument : «je l'ai dit à Olivier Faure, c'est une très mauvaise idée d'aller dans une ville communiste où le PS siège dans l'opposition et refuse l'union de la gauche. Qu'on fasse un peu

16. Également financièrement fragilisé par ses résultats électoraux, LR s'est engagé dans une autre voie : vendre son siège, sans pour autant déménager, et rester locataire. 
de politique quand même!» (Le Monde, le 12 mai 2018). Philippe Bouyssou, le maire communiste d'Ivry-sur-Seine, prend le parti d'ironiser sur la décision du PS de se porter sur sa ville : «je suis étonné que les socialistes n'aient pas trouvé une ville plus emblématique de la gestion locale du PS. Cela montre que ce que nous faisons n'est pas si mal».

La motivation économique a ensuite été troquée contre une logique plus spatiale, soulignant ainsi que les toponymes appartiennent également à des «couches de population » (Rostaing, 1965, p. 5). Répondant à ses détracteurs, 0 . Faure fait alors le choix de politiser la disparition de cette substitution métonymique, rappelant que le choix d'un lieu pouvait « concourir à socialiser politiquement “leurs” électeurs» (Sawicki, 1996) : «ma préférence [pour le siège] va vers Ivry-sur-Seine, une façon de rompre avec le passé et le symbole d'un PS qui vient retrouver ses racines populaires» (CNews, le 2 mai 2018). En quittant un hôtel particulier et le $7^{\mathrm{e}}$ arrondissement pour rejoindre une ancienne manufacture et la banlieue parisienne, le PS envoie un signe politique à un électorat qui l'avait quitté depuis de nombreuses années (Lefebvre, Sawicki, 2006) : «à Solférino, on avait un bâtiment prestigieux. Ici, nous sommes de plain-pied dans la vie, y compris avec les gens que nous souhaitons représenter ${ }^{17}$. De son côté, S. Le Foll a refusé de se plier à cet exercice de sociotoponymie (selon le terme de Boyer, 2008) : «est-ce qu'on a perdu des élections quand on était à Solférino? Comment peut-on arriver à considérer que le fait de changer de lieu change la politique?»(CNews, 18 mai 2018).

Au-delà du statut politique de la ville d'Ivry-sur-Seine, c'est in fine la géographie même de cette commune qui a été ciblée par les opposants de la direction du PS. La décision de quitter la rue de Solférino et sa proximité avec l'Assemblée nationale pour une ville de banlieue - qui plus est communiste - a été ressentie comme un signe de déclassement du PS. E. Maurel, représentant de l'aile gauche du PS, déclare ainsi : «là, au moins c'était central, un parti de gouvernement doit être dans la capitale». PourP. Kanner, «il ne fallait pas quitter Paris, nous ne sommes pas un groupuscule. Un siège, c'est important en termes d'image» (Le Monde, le 4 mai 2018).

Le maintien de la siglaison socialiste n'a donc pas circonscrit l'espace de discussion. Comme le soulignent Paul Bacot, Dominique Desmarchelier et JeanPaul Honoré, les réductions comme les symboles toponymiques font « office de signature» (Bacot, Desmarchelier, Honoré éd., 2011, p. 7). La «bataille de Solférino » livrée en parallèle du congrès d'Aubervilliers a soumis les responsables du PS à un examen qui excède l'argument économique de la vente d'un simple bâtiment. À cette narration univoque se sont rapidement opposés des récits qui n'avaient pas trouvé leur place pendant la phase officielle des motions et des candidatures au poste de premier secrétaire. En décidant de vendre leur

17. Sur l’identification entre représentants et représentés, voir Déloye, 2017. 
siège, les responsables socialistes ont changé d'adresse à défaut de nom, non sans difficultés organisationnelles ${ }^{18}$, et ainsi symbolisé la défaite électorale de leur parti, chargeant de sens une dénomination toponymique qui correspondait à un « ancrage anthropologique » (Sawicki, 1996) peu conforme à l'image politique souhaitée par la nouvelle direction du PS.

L'abrègement typographique socialiste n'a donc pas été changé. Malgré l'inscription de cet enjeu lors du congrès d'Aubervilliers de 2018 et les ruptures onomastiques déjà engagées par une grande partie des formations politiques françaises, le PS a gardé sa siglaison. Les défaites électorales de 2017 n’ont pas été génératrices d'injonctions au changement nominatif, même formulées de manière routinisée. Atteint par le résultat de $B$. Hamon à l'élection présidentielle, miné par les départs des deux finalistes de sa primaire et encombré par l'omniprésence médiatique de F. Hollande ${ }^{19}$, le PS a fait le choix de la fidélité à l'un de ses derniers brevets d'authenticité. Marqué par l'épuisement de la «grammaire de l'institution partisane socialiste » de gestion de ses crises (Lefebvre, 2018), le PS a préféré dire "adieu à Solférino »20 et perdre un substitut discursif symbolique, trouvant ainsi un autre moyen communicationnel d'imputation de l'échec politique que de se voir confronté à une nouvelle réduction, la permanence dans le sigle PS suffisant alors pour ses responsables «pour attester, dans la durée, l'existence d'un Parti socialiste français » (Offerlé, 2018 [1987], p. 117).

\section{Références}

ALDRIN Philippe, 2007, «Si près, si loin du politique : l'univers professionnel des permanents socialistes à l'épreuve de la managérialisation », Politix, $n^{\circ} 79$, p. 25-52.

BACOT Paul, 2018a, «Onomastique partisane», dans Rencontres avec Michel Offerlé, H. Michel, S. Lévêque et J.-G. Contamin éd., Vulaines-sur-Seine, Éditions du Croquant.

- 2018b, «Ce n'est pas un départ, c'est une scission», billet sur le site de la Société d'étude du langage du politique, https://selp.eu/figures/ce-nest-pas-un-departcest-une-scission/ (consulté le 10 mars 2019).

- 2010, «Développement et diversification d'une onomastique politique », Mots. Les langages du politique, nº 94, p. 47-56.

18. La direction du PS a dû renoncer à trois reprises à l'inauguration de ses locaux d'Ivry-sur-Seine, en raison notamment du mouvement des «gilets jaunes».

19. François Hollande a sorti son livre Les leçons du pouvoir le mercredi 11 avril 2018, dans la phase finale du congrès d'Aubervilliers, perturbant ainsi fortement l'expression du nouveau premier secrétaire.

20. L'Adieu à Solférino, documentaire de Grégoire Biseau et Cyril Leuthy diffusé sur Public Sénat le samedi 16 février 2019. 
Bacot Paul, Desmarchelier Dominique, Honoré Jean-Paul éd., 2011, Sigles et acronymes en politique [numéro thématique], Mots. Les langages du politique, $\mathrm{n}^{\circ} 95$.

BARbonI Thierry, TREILle Éric, 2010, "L'engagement 2.0: les nouveaux liens militants au sein de l'e-parti socialiste», Revue française de science politique, vol. LX, nº 6 , p. 1137-1157.

BOYER Henri, 2008, «Fonctionnements sociolinguistiques de la dénomination toponymique », Mots. Les langages du politique, n08, p. 9-21.

CAmbadÉlıS Jean-Christophe, 2016, Chronique d'une débâcle : 2012-2017, Paris, L'Archipel.

CÉPÈde Frédéric, 2017, «Le nom du Parti socialiste : dire ce que l'on est (1905-1971)», L'Ours, 5 septembre, https://www.lours.org/le-nom-du-parti-socialiste-dire-ce-quelon-est-1905-1971/.

- 1996, "“Le poing et la rose”, la saga d'un logo », Vingtième Siècle. Revue d'histoire, $\mathrm{n}^{\circ} 49$, p. $18-30$.

- 1994, "Les insignes du Parti », Cahier et Revue de l'Ours, n²04.

CÉPÈdE Frédéric, ALMEIDA Fabrice d', 2007, «Être socialiste d'un siècle à l'autre : la tradition militante à l'épreuve des logiques médiatiques», Vingtième siècle. Revue d'histoire, nº 96, p. 91-105.

Cos Rafaël, 2016, «Le projet socialiste (dés)saisi par les primaires : procédures “rénovatrices" et production programmatique », dans Les primaires ouvertes en France : adoption, codification, mobilisation, R. Lefebvre et É. Treille éd., Rennes, Presses universitaires de Rennes, p.163-180.

DÉLOYE Yves, 2017, «Se présenter pour représenter. Enquête sur les professions de foi électorales de 1848 ", dans La profession politique : XIXe-xxe siècles, M. Offerlé éd., Paris, Belin.

Dolez Bernard, Fretel Julien et LefebVRe Rémi éd., 2019, L'entreprise Macron, Fontaine, Presses universitaires de Grenoble.

DORMAGEN Jean-Yves, 1995, "Au nom du nom : la fin du parti communiste italien et la naissance du parti de la refondation communiste », Vingtième Siècle. Revue d'histoire, $n^{\circ} 48$, p. 75 -90.

Ethuin Nathalie, Lefebvre Rémi, 2015, "Réinventer les partis politiques? », Savoir/ Agir, $\mathrm{n}^{0}$ 32, p. 9-12.

Fiala Pierre, Rennes Juliette, 2002, "Majorité plurielle, trajectoire d'une formule», Mots. Les langages du politique, $\mathrm{n}^{\circ} 68, \mathrm{p} .123-130$.

GUILLOREL Hervé, 2012, "Onomastique, marqueurs identitaires et plurilinguisme. Les enjeux politiques de la toponymie et de l'anthroponymie », Droit et cultures, nº 64 , p.11-50.

HAEGEL Florence, 2002, "Faire l'union : la refondation des partis de droite après les élections de 2002 ", Revue française de science politique, vol. LII, nº 5-6, p. 561-576.

LAZAR Marc, 1992, Maisons rouges : les Partis communistes français et italien de la Libération à nos jours, Paris, Aubier.

LeCA Jean, Offerté Michel, 1988, «Un “Que sais-je?” en questions », Politix, n² 2, p. 46-59. LefEBVRe Rémi, 2018, Après la défaite : analyse critique de la rénovation au Parti socialiste, 2002-2007-2017, Paris, Fondation Jean Jaurès. 
- 2017, " “Dépassement” ou effacement du Parti socialiste (2012-2017) ?», Mouvements, n०89, p.11-21.

LefebVRe Rémi, SAWICKı Frédéric, 2006, La société des socialistes : le PS aujourd'hui, Bellecombe-en-Bauges, Éditions du Croquant.

Lefebvre Rémi, Treille Éric éd., 2019, Les primaires ouvertes : un nouveau standard international ?, Villeneuve-d'Ascq, Presses universitaires du Septentrion.

- 2018, «Le déclenchement des primaires ouvertes chez Les Républicains et au Parti socialiste : entre poids du précédent de 2011 et bricolages organisationnels (20162017) », Revue française de science politique, vol. LXVII, nº 6, p.1167-1185.

- 2016, Les primaires ouvertes en France : adoption, codification, mobilisation, Rennes, Presses universitaires de Rennes.

OfFERLÉ Michel, 2018 [1987], Les partis politiques, Paris, PUF.

- 2017, «Les professionnalisations politiques en question(s)», postface à La profession politique : $x x^{e}-x x I^{e}$ siècles, M. Offerlé éd., Paris, Belin.

Perrineau Pascal éd., 2017, Le vote disruptif : les élections présidentielles et législatives de 2017, Paris, Presses de Sciences Po.

Phélippeau Éric, 2018, L'argent de la politique, Paris, Presses de Sciences Po.

PUDAL Bernard, 2009, Un monde défait : les communistes français de 1956 à nos jours, Bellecombe-en-Bauges, Éditions du Croquant.

- 2002, "La beauté de la mort communiste», Revue française de science politique, vol. LII, nº 5-6, p. 545-559.

RIOUFREYT Thibaut, 2016, Les socialistes français face à la troisième voie britannique : vers un social-libéralisme à la française (1997-2015), Fontaine, Presses universitaires de Grenoble.

Rostaing Charles, 1965, Les noms de lieux, Paris, PUF.

SAWICKI Frédéric, 2017, "L'épreuve du pouvoir est-elle vouée à être fatale au Parti socialiste? Retour sur le quinquennat de François Hollande », Pouvoirs, nº 163, p. 27-41.

- 1996, "La science politique et l'étude des partis politiques», Cahiers français, $n^{\circ} 276$, p. 51-59.

TOURNIER Maurice, 1981, «Vers une grammaire des dénominations socio-politiques au début de la Troisième République (1879-1905)», Mots, n² 2, p. 51-72.

TREILLE Éric, 2019, "C'est aussi notre créature”. Emmanuel Macron et le PS entre logiques de positionnement et instrumentalisation tactique», dans L'entreprise Macron, B. Dolez, J. Fretel et R. Lefebvre éd., Fontaine, Presses universitaires de Grenoble, p. 27-38.

— 2018, «L'économie de la démocratie partisane : la réglementation financière de la vie politique à l'épreuve des primaires de 2016-2017 », dans Financement et moralisation de la vie politique, E. Forey, A. Granero et A. Meyer éd., Bayonne, Institut universitaire Varenne.

- 2001, «Littérature de campagne. Objets de propagande et élections législatives», dans L'implantation du socialisme en France au XXe siècle : partis, réseaux, mobilisation, J. Girault éd., Paris, Publications de la Sorbonne, p. 323-334. 


\section{Résumé / Abstract / Compendio}

\section{Fin de parti(e). La dénomination du PS en questions}

Malgré l'inscription de l'enjeu de son abrègement typographique lors du congrès d'Aubervilliers de 2018 et les ruptures onomastiques déjà connues par une grande partie des formations politiques françaises, le PS a conservé sa siglaison. Parti fragilisé par le résultat historiquement faible de son candidat à l'élection présidentielle de 2017, il a fait le choix de la fidélité à l'un des éléments les plus forts de son identité, tout en trouvant d'autres voies symboliques pour sortir de l'après-défaite, comme la vente de son siège rue de Solférino et la perte d'un substitut discursif emblématique.

Mots-clés : Parti socialiste, primaire, onomastique, sigle partisan

\section{End of (the) party. The name of the PS in questions}

Despite the inscription of the challenge of its typographical abolition at the Aubervilliers congress of 2018 and the onomastic breaks with the past that are familiar to many of the French political parties, the Socialist party has retained its siglaison. The party, which was weakened by the historically poor result of its candidate in the 2017 presidential election, chose loyalty to one of the strongest elements of its identity, while finding other symbolic ways of leaving electoral defeat behind, with for example the sale of its headquarters in the rue de Solferino and the loss of an emblematic discursive substitute.

Keywords: French socialist party, primary, onomastics, partisan acronym

\section{Fin del partido. El nombre del PS en cuestión}

A pesar de la inscripción del desafío de su abolición tipográfica en el congreso de Aubervilliers de 2018 y las rupturas onomásticas ya conocidas por una gran parte de los partidos políticos franceses, el Partido Socialista francés ha conservado su acrónimo. Partido debilitado por el resultado de históricamente frágil de su candidato a las elecciones presidenciales de 2017 , eligió la lealtad a uno de los elementos más fuertes de su identidad al mismo tiempo que encontraba otras vías simbólicas para salir de la post derrota, como la venta de su sede en la calle de Solferino y la pérdida de un sustituto discursivo emblemático.

Palabras claves: Partido Socialista francés, primario, onomástica, acrónimo partidista 